Kalpa Publications in Engineering
Volume 3, 2020, Pages 154-162
Proceedings of International Sym-
posium on Applied Science 2019

\title{
In Silico Assessment Of The Impedance Parameters Of Tissue Using A 3D Finite Element Model To Detect Breast Cancer
}

\author{
Son Thuy Nguyen Nhu ${ }^{1}$, Quang Linh Huynh ${ }^{1}$ \\ ${ }^{1}$ Ho Chi Minh City University of Technology - VNUHCM \\ nnsonthuy@hcmut.edu.vn
}

\begin{abstract}
The early diagnosis benefits the greater efficiency of the curing of breast cancer. One of the modalities for detection of abnormal tissue in present days is electrical impedance myography (EIM). EIM is a noninvasive electrophysiological technique, using high frequency, low-intensity electrical current to derive voltage at the positions of sense electrodes. A Comsol Multiphysics-based finite element model was carried out in order to build 3D model of breast tissue for computational simulation of mentioned technique. EIM parameters of breast tissue involving resistance, reactance, phase angle in case of malignant tissue were calculated and compared with parameters of normal tissue. The results of the study showed some remarkable effects of tumor sizes, tumor positions and electrode positions on the EIM parameters, which can be considered for potential early detection.
\end{abstract}

\section{Introduction}

Breast cancer is the second most common cause of death from the cancer in women in the United States, after lung cancer. If the cancer is located only in the breast, the 5year survival rate of women with breast cancer is $99 \%$ (62\% of people with breast cancer are diagnosed with this satge). If cancer has spread to the regional lymph nodes, the 5year survival rate is $85 \%$. If the cancer has spread to a distant part of the body, the 5- year survival rate is $27 \%$ [5]. It means that the early detection significantly help to increase the survival rate and treatment effect. In present days, there are three different imaging techniques, i.e., ultrasound, magnetic resonance imaging and X-ray. However, they are ineffective, expensive and at the risk of tumor development. In comparison to these three methods, Electrical Impedance Myography (EIM) has profound advantages [1].

EIM is non- invasive, painless electrophysiological approach using high frequency and low intensity electrical current. Low amplitude electrical current is applied via two outer electrodes and the corresponding voltage patterns are analyzed by two inner electrodes. EIM assesses three major 
parameters: resistance, reactance and phase [3]. The value of these parameters depends not only on the tissue condition but also the position of electrodes.

The complex impedance can be expressed mathematically:

$\mathrm{Z}=\mathrm{R}+\mathrm{jX}$ (1).

Where $Z(\Omega)$ is the impedance, $R(\Omega)$ is the resistance, $X(\Omega)$ is the reactance.

The Ohm's law:

$\mathrm{V}=\mathrm{IZ} \quad(2)$

Where $\mathrm{V}(\mathrm{V})$ is the voltage.

So, the complex admittance becomes:

$Y=\frac{1}{z}=G+j \omega C(3)$

Where $\mathrm{G}$ is the conductance and capacitance is $\mathrm{C}$.

Here $G=\frac{R}{R^{2}+X^{2}}(4)$ and $C=\frac{X}{\left(R^{2}+X^{2}\right) w}(5)$

So the muscles internal electrical property, conductivity and relative permittivity depend on conductance, capacitance and geometric factor. So the conductivity, $\sigma=K . G$ (6)

And the relative permittivity $\varepsilon_{r}=\frac{K . C}{\varepsilon}(7)$, where $K=\frac{d}{A}$ is the geometry factor of muscle. Here $\mathrm{d}$ is the distance between voltage electrodes and A is cross sectional area of the muscle [3].

\section{Simulation Method}

The objective of this study is to investigate the effect of tumor size, the position of tumor, the position of electrodes on the EIM parameters. In order to do that, Comsol Multiphysics software and finite element model was applied.

Finite Element Method is a numerical approach for obtaining the solutions to physical realityformulated in a mathematical model by splitting the boundaries into smaller, simpler parts. In our study, electrical current physics interface is used to compute the electric field and potential distribution in conducting tissue under frequency domain analysis. By using this physics interface, current conservation equation is solved based on Ohm's law using the scalar electric potential as the dependent variable [1]. The solved equation is given.
$\nabla . \mathrm{J}=\mathrm{Q}_{\mathrm{j}}$
$\mathrm{J}=\left(\sigma+\mathrm{j} \omega \varepsilon_{0} \varepsilon_{\mathrm{r}}\right)+\mathrm{J}_{\mathrm{e}}(9)$
$\mathrm{E}=-\nabla \mathrm{V}$ 


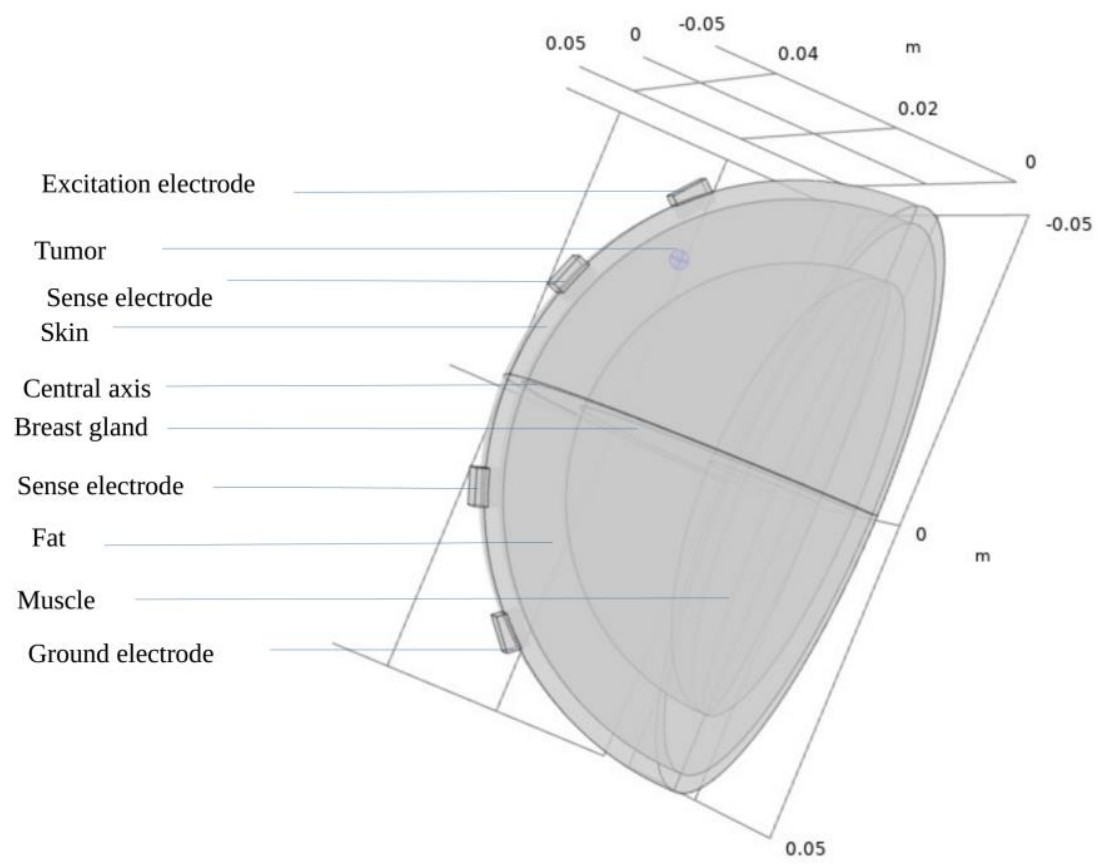

Figure 1. 3D model of breast tissue

Where Qj is Boundary current source which is applied in the boundary surface of the excitation electrode and $\mathrm{E}$ is the electric field which depends on dependent variable $\mathrm{V}$ and $\mathrm{J}$ is the total current density [1].

Breast cancer is constructed as 3D model involving four layers: skin, breast fat, gland tissue, muscle. Tumor tissue has spherical shape and is placed in the middle of fat. Four square electrodes with $0.6 \mathrm{x}$ $0.6 \mathrm{~cm} 2$ of area are kept $17 \mathrm{~mm}-30 \mathrm{~mm}-17 \mathrm{~mm}$ which is measured from edge to edge. The conductivity and permittivity of tissue are obtained from published source [4]. Assuming that electrodes are perfect conductor, the conductivity and permittivity of electrodes are set to $5.0 \mathrm{e} 5 \mathrm{~S} / \mathrm{m}$ and 1.0 respectively. According to EIM, a high frequency $2.45 \mathrm{GHz}$ is applied and a small current source of $1 \mathrm{~mA}$ is supplied from excitation electrode while voltage is measured from sense electrodes

\section{Results and Discussions}

\subsection{Examination the effect of tumor size}

Firstly, we examined the effect of tumor size on three EIM parameters: resistance, reactance and phase. The distances of electrodes were kept $17 \mathrm{~mm}-30 \mathrm{~mm}-17 \mathrm{~mm}$. Tumor was located in breast fat, underneath the area of electrodes. Moreover, tumor size varied from $2 \mathrm{~mm}$ to $8 \mathrm{~mm}$. In stage one of breast cancer, the size of tumor is smaller than $20 \mathrm{~mm}$. Therefore, in this study, the size of tumor was set smaller than $20 \mathrm{~mm}$ to improve the ability of early detection breast cancer. 


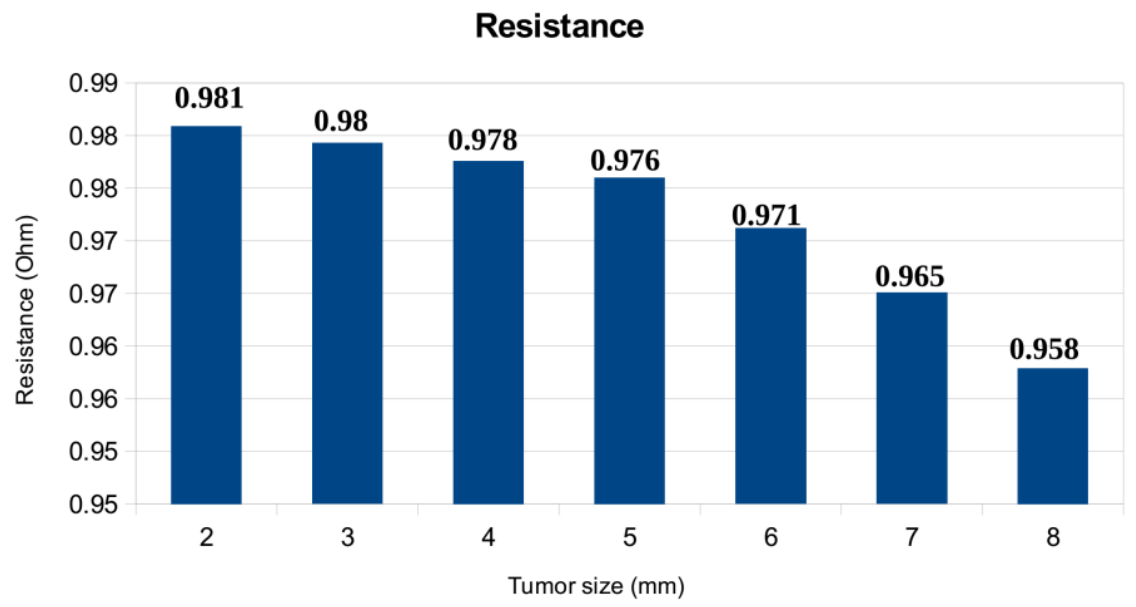

Figure 2. Resistance with respect to tumor size

The simulated resistance value of normal breast tissue was found to be 0.981 . Considering breast tumor as processing simulation, while tumor size increased, the resistance values decreased slightly and fell furthermore for the tumor size of 6- $8 \mathrm{~mm}$. The percentage deviation of resistance value was $1.03 \%$ at $6 \mathrm{~mm}$ and $2.4 \%$ at $8 \mathrm{~mm}$.

It can be interpreted that conductivity and permittivity of tumor are greater than the ones of other tissue. Thus, as tumor size increased, the current going through the tissue rose, then resistance values reduced.

Interestingly, the reactance not only reduced when tumor size increased, but also appeared to be more affected in comparison with the resistance. It is clear from Figure 3 that reactance started at 4.28 $\Omega$ from $2 \mathrm{~mm}$ tumor, then it continued to drop until it reached to $4.176 \Omega$ for $8 \mathrm{~mm}$ tumor. As comparison to the reactance of normal breast with $4.28 \Omega$ of value, the percentage deviation as $0.96 \%$ and $2.49 \%$ for $6 \mathrm{~mm}$ and $8 \mathrm{~mm}$ tumor respectively.

We noted that in biological systems, resistance is caused by the material of outer and inner tissue while reactance occurs due to the capacitance of the cell membrane. The increase of tumor size led to the jump of conductivity and permittivity, as a consequence, resistance and reactance went down. However, in EIM method, due to high frequency, current could easily go across cell membranes into inner tissue, thus reactance appeared to decrease greater than resistance.

In addition, we also considered the change of phase according to the rise of tumor size. According to the Figure 4, there was no significant change of phase when tumor became larger. It is worthwhile to note that during the increase of tumor size, resistance and reactance values reduced. These results are not only quite similar to the work of Tarek et al [1] but also meaningful for he detection of breast cancer. 


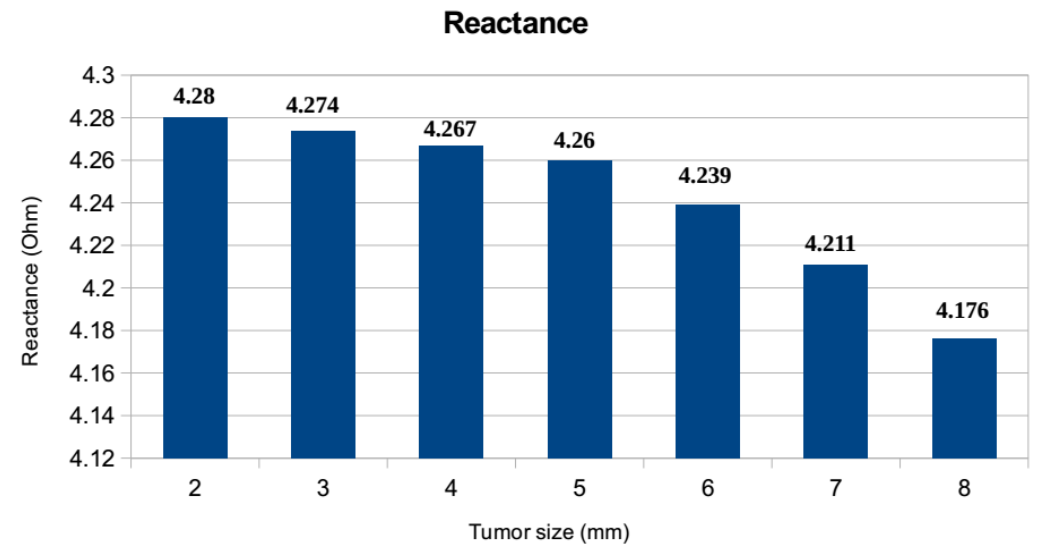

Figure 3. Reactance with respect to tumor size

Phase

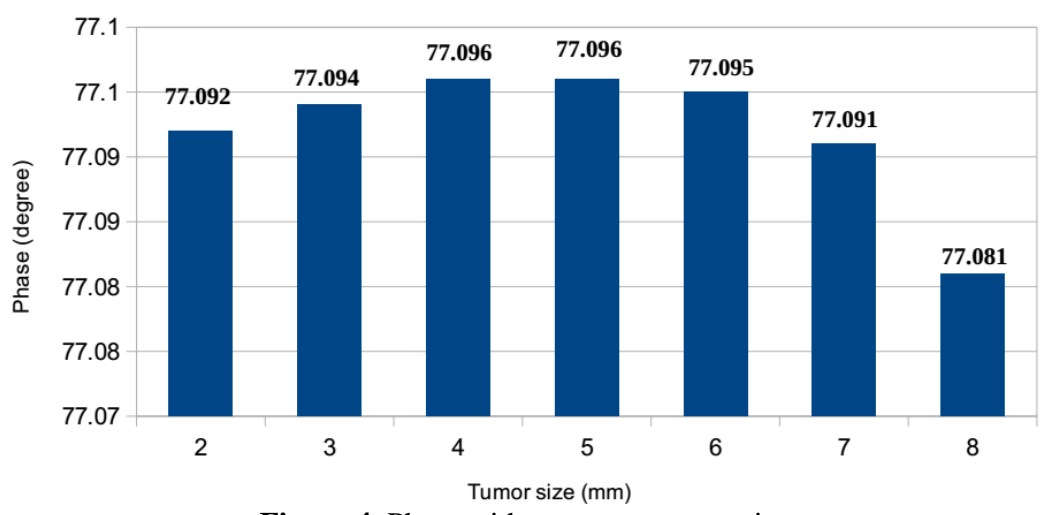

Figure 4. Phase with respect to tumor size

\subsection{Examination the correlation of electrode position and tumor position with impedance value}

To have deeper study of electrode position, we first looked into the effect of electrode position and tumor position on impedance value.

Four electrodes were kept $17 \mathrm{~mm}-30 \mathrm{~mm}-17 \mathrm{~mm}$ symmetrically around the central axis. $3 \mathrm{~mm}$ tumor was placed in breast fat. While the electrodes were kept at fixed position the angle of tumor was altered from 10 to 85 degree related to the central axis.

We found that when the tumor was underneath the four electrodes (tumor angle smaller than 60 degree), the impedance values were slightly smaller than the ones when tumor was outside the region of electrodes. The figure shows that when tumor angle was smaller than 60 degree, the impedance values varied around $4.38 \Omega$ while the impedance value grew and reached to the highest point of value reduces as compared to the situation of outside the $4.395 \Omega$ when tumor angle equal or greater than 60 degree. electrode zone.

It can be considered that when tumor is in the position of electrodes, current density increases, thus the impedance. 


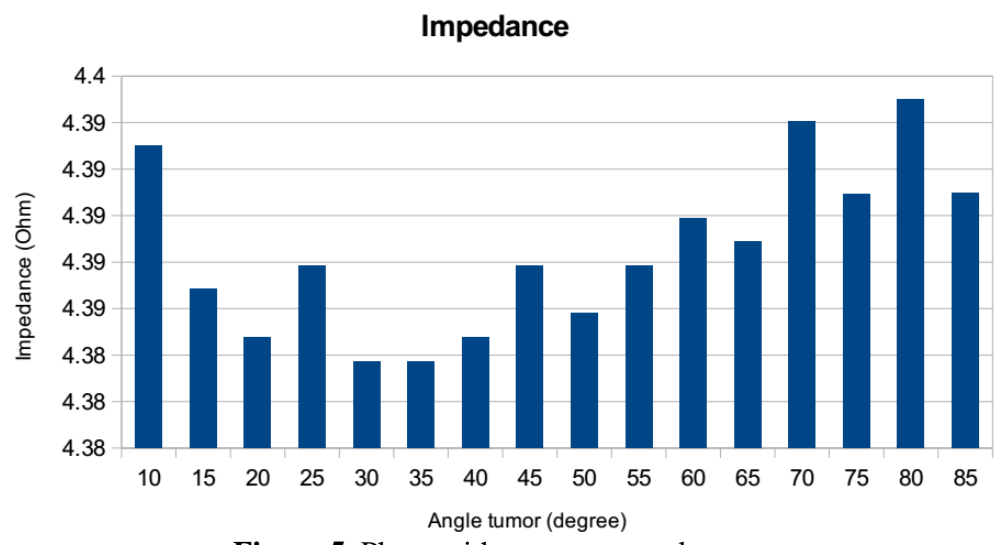

Figure 5. Phase with respect to angle tumor

\subsection{Specification the effect of electrode position}

To shed light on how electrode position affects the impedance value, we examined electrode position via two situations: tumor underneath electrode region and tumor outside electrode zone.

\subsubsection{Tumor underneath electrode region}

Tumor with $3 \mathrm{~mm}$ diameter was kept at fixed position, in breast fat, with 40 degree relative to the central axis.

Excitation and ground electrodes were 50 degree axially symmetric around the central axis. While sense electrodes varied their angles from 40 degree to 10 degree related to the central axis, they were also kept axially symmetric.

Interestingly, while sense electrodes moved further from excitation and ground eletrodes (from 40 to 10 degree), impedance values reduced from $13.205 \Omega$ to $1.460 \Omega$.

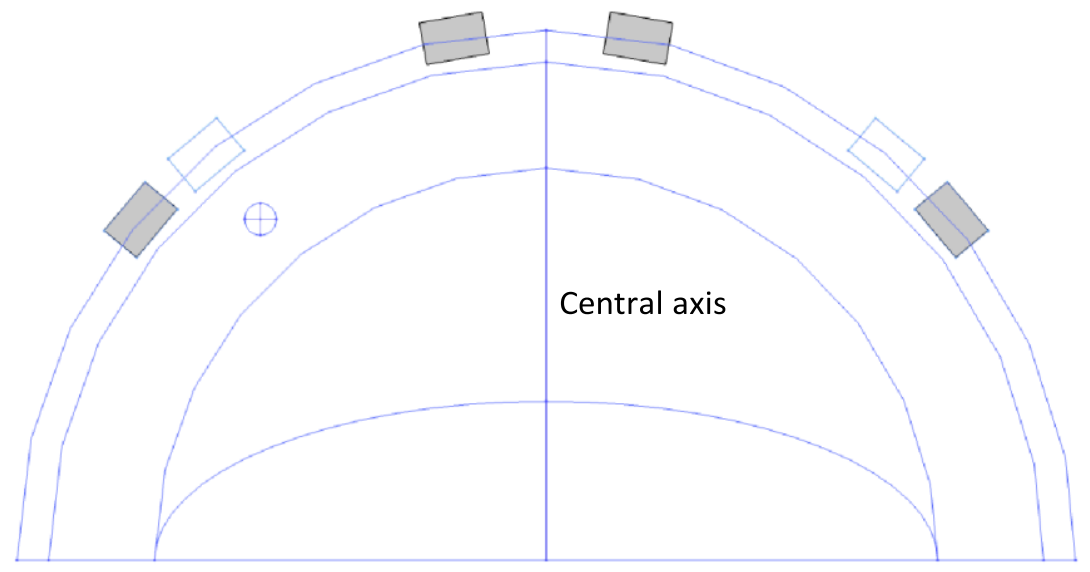

Figure 6. Tumor underneath electrode region 


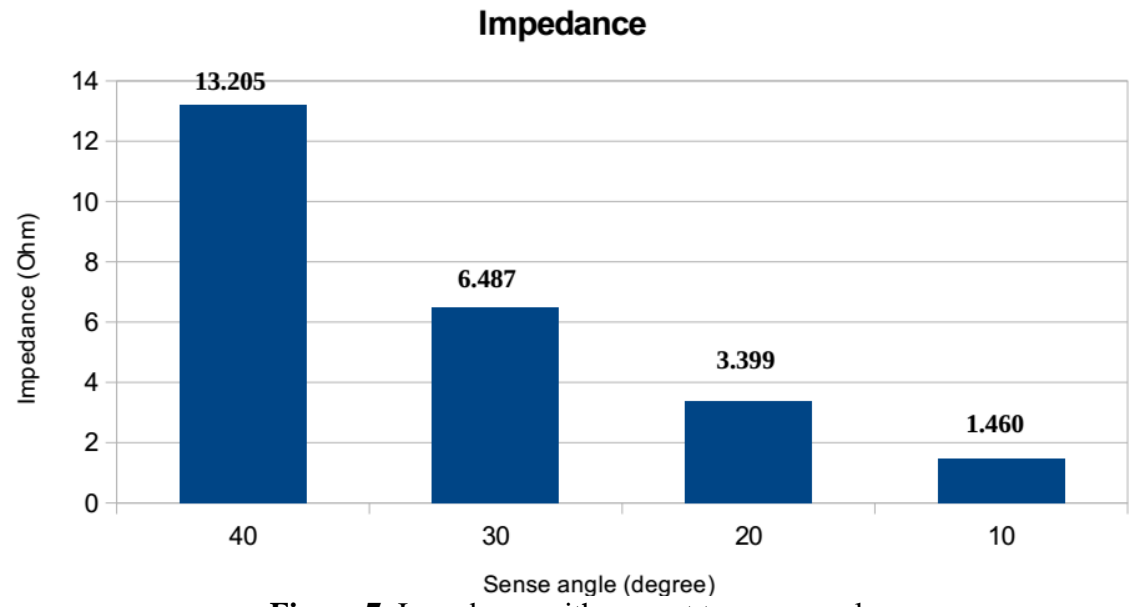

Figure 7. Impedance with respect to sense angle

\subsubsection{Tumor outside electrode zone}

Tumor with $3 \mathrm{~mm}$ diameter was kept at fixed position, in breast fat, with 60 degree relative to the central axis. Excitation and ground electrodes were 50 degree axially symmetric around the central axis. While sense electrodes varied their angles from 40 degree to 10 degree related to the central axis, they were also kept axially symmetric.

Similarly to the previous situation, while sense electrodes moved further from excitation and ground eletrodes (from 40 to 10 degree), impedance values reduced from $13.228 \Omega$ to $1.462 \Omega$, slightly greater than the previous situation. It is worthwhile to note that when sense electrodes moved farther from excitation and ground electrodes, the impedance values went down.

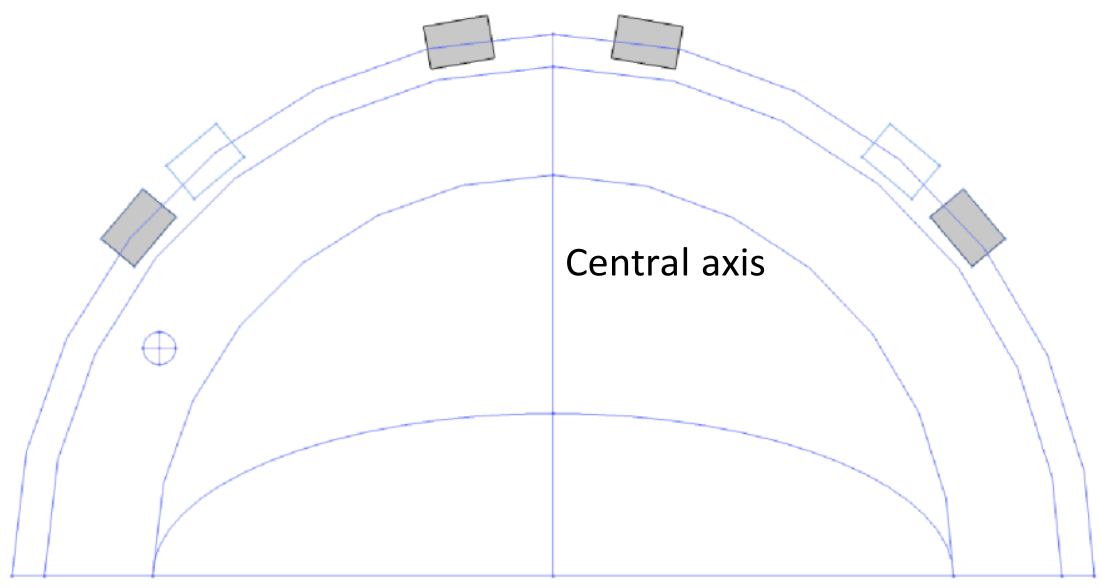

Figure 8. Tumor outside electrode zone 


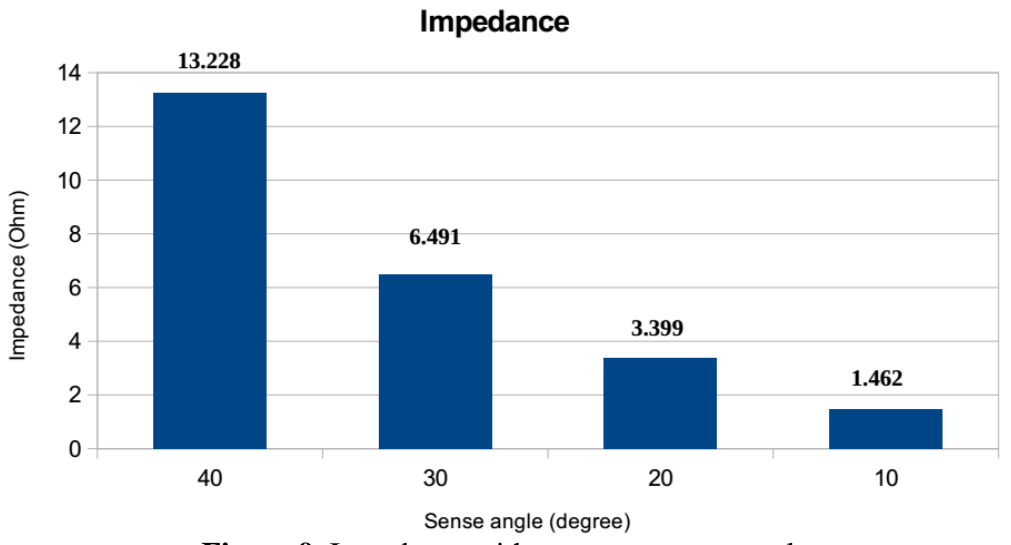

Figure 9. Impedance with respect to sense angle

\subsection{Tumor at different tissue layers}

Breast cancer is classified into several stages according to tumor size and their spreading position. Therefore, it is meaningful to consider the effect of tumor position in different tissue layers on the impedance value.

The model involved a $3 \mathrm{~mm}$ tumor, with 40 degree relative to the central axis. Electrodes were kept at fixed distance $17 \mathrm{~mm}-30 \mathrm{~mm}-17 \mathrm{~mm}$. While the tumor angle with the central axis was unchanged, the malignant tissue was moved through layers: breast fat, gland tissue and muscle.

We found that while tumor moved from breast fat to gland tissue, the impedance values jumped from $4.385 \Omega$ to $4.392 \Omega$. However, the impedance values remained constant while the tumor moved from gland tissue to muscle.

It can be explained that when the tumor moved deeper into layers, current density decreased, thus the impedance value increased. Understanding the effect of tumor position in different layers gives a hint for early detection breast cancer.

\section{Conclusion}

To summarize this work, we found that tumor position and electrode position have effect on the EIM parameters and reactance appears to be more affected in comparison with the resistance and phase. Moreover, when tumor is underneath the electrode range, the impedance values are smaller than the ones when tumor is outside the region of electrodes. Furthermore, the impedance values will decrease when sense electrodes move farther excitation and ground electrodes. This interesting result gives hint for researches optimizing the electrode position on bioimpedance measurement. Another significant result is that the impedance values will also reduce while tumor moves deeper into tissue layers. This practical result benefits for further researches on early detection of breast cancer.

For further work, we will optimize the distribution of electrodes to detect and determine tumor position for the goal of breast cancer detection. 


\section{References}

Tarek M. N., Jalal A. H., Alam F., Ahad M. A. Detection of the breast cancer based on the electrical impedance myography parameters using finite element method, Smart biomedical and physiological sensor technology- 15 (106620I) (2018).

Shash Y. H., Eldosoky M. A. A., Elwakad M. T. - The effect of vascular diseases on bioimpedance measurements: mathematical modeling, Biomedical research and therapy- 5 (6) (2018) 2414-2431.

Rabbi K. M. F. - Assessment of electrode configurations of Electrical impedance myography for the evaluation of neuromuscular diseases, Rabbi K. M. F. - Assessment of electrode configurations of Electrical impedance myography for the evaluation of neuromuscular diseases, Dissertations, Spring (2015) 1273.

https://www.itis.ethz.ch/virtual-population/tissueproperties/database/dielectric-properties.

https://www.cancer.net/cancer-types/breast-cancer/Dissertations, Spring (2015) 1273. stages 